\title{
Resolución de traumatismo por mordedura de perro en tortuga argentina (Chelonoidis chilensis) mediante aproximación de piel y utilización de miel de abeja
}

\author{
Dog bite trauma resolution in tortoise (Chelonoidis \\ chilensis) by skin approximation and use of honey
}

\author{
Vetter Hiebert, Joerg Richard; González Vatteone, Roger; Fretes Isasi, Axel \\ Manuel
}

\section{Joerg Richard Vetter Hiebert}

Departamento de Recursos Faunísticos y Medio Natural, Paraguay

jvetter@vet.una.py

\section{Roger González Vatteone}

Cátedra de Técnica Operatoria, Paraguay

\section{Axel Manuel Fretes Isasi}

Estudiante. Facultad de Ciencias Veterinarias. Universidad Nacional de Asunción, República del Paraguay, Paraguay

\section{ANALECTA VETERINARIA \\ Universidad Nacional de La Plata, Argentina ISSN: $1514-2590$ \\ Periodicidad: Semestral \\ vol.41, núm.2,e058, 2021 \\ analecta@fcv.unlp.edu.ar}

Recepción: 19 Agosto 2021

Revisado: 30 Agosto 2021

Aprobación: 11 Noviembre 2021

URL:

http://portal.amelica.org/ameli/jatsRepo/25/2524400 02/ index.html

DOI:https://doi.org/10.24215/15142590e058

\section{(i)(2)}

Esta obra está bajo una Licencia Creative Commons Atribución- NoComercial-CompartirIgual 4.0 Internacional.
Resumen: Se describe el caso de un ejemplar de tortuga argentina, Chelonoidis chilensis, macho, adulto, que fue atacado y mordido por un canino. Se constató la fractura del plastrón, con pérdida del segmento frontal y exposición de los músculos deltoideus y supracoracoideus. Se fijaron 3 ganchos al plastrón mediante resina epoxi, aproximando la piel a los mismos con poliamida o,30. Se utilizó cefazolina como tratamiento antibiótico y los espacios abiertos entre piel y plastrón fueron cubiertos con miel de abeja en forma tópica cada 48 horas. Al día 5 de tratamiento se percibió el crecimiento de Fusarium spp. en la superficie, por lo que se modificó el tratamiento local y se procedió a realizar diariamente el lavado de la zona con iodopovidona al 7,5 \%, seguido de la aplicación de miel. Se realizaron baños en agua durante 2 horas, cada 48 horas. A los 5 días del procedimiento, el paciente comenzó a ingerir agua y, a los 35, a alimentarse. A los 28 días se percibió buena adherencia de la piel a los músculos, al tiempo que, coincidentemente, empezó a utilizar los miembros anteriores. A las 6 semanas se retiraron las suturas y se constató remodelación del tegumento y cierre completo de la herida.

Palabras clave: tortuga argentina, Chelonoidis chilensis, miel de abeja, traumatismo.

\begin{abstract}
A case of a male, adult tortoise (Chelonoidis chilensis) attacked by a dog is described. The animal presented a fractured plastron, with loss of the frontal segment, exposing the deltoideus and supracoracoideus muscles. Three hooks were fixed to the plastron with epoxy resin, approximating the skin to them with polyamide 0.30. Antibiotic treatment consisted in cefazoline, and the open spaces between skin and plastron were covered with honey every 48 hours. Growth of Fusarium spp. was detected at the $5^{\text {th }}$ day on the surface treated with honey, therefore the topical treatment was modified by adding a daily washing with $7.5 \%$ povidone-iodine prior to the application of honey. In addition, water baths were performed for 2 hours, every 48 hours. The patient started drinking water and eating food at the $5^{\text {th }}$ and $35^{\text {th }}$ day, respectively. Good adherence of the skin to the muscles was observed at 28 days when, coincidentally, the patient began using the forelimbs. At 6 weeks, the sutures were removed, and a remodeling of the integument and a complete closure of the wound were observed.
\end{abstract}

Keywords: Argentine tortoise, Chelonoidis chilensis, honey, trauma 


\section{Introducción}

La tortuga terrestre argentina, Chelonoidis chilensis (Gray, 1870), se distribuye ampliamente a través del territorio argentino y paraguayo, al igual que en el sur de Bolivia (van Dijk et al, 2014).

El plastrón de las $C$. chilensis está dividido en lóbulos anterior y posterior. Estos lóbulos se subdividen en escudos, a saber: gulares, humerales, pectorales, abdominales, femorales, anales, axilares, inguinales (Cabrera, 1998).

Las tortugas frecuentemente presentan lesiones en el caparazón, debido a diversos motivos como, por ejemplo, traumatismos producidos por cortadoras de césped, vehículos o mordeduras de depredadores, ya sea perros, zorros o ratas (Paterson, 2006; Pereira Dutra, 2014; Romero et al, 2019; Vetter \& Riveros, 2021). En Paraguay, de todos los pacientes quelonios que se presentaron entre los años 2015 y 2019 en el Consultorio de Animales Silvestres y Exóticos, dependiente del Departamento de Recursos Faunísticos y Medio Natural de la Facultad de Ciencias Veterinarias de la Universidad Nacional de Asunción, el 8,4 \% acudió por traumatismos debido a ataques de perros domésticos (Quintana, comunicación personal).

La miel de abeja ha sido utilizada ampliamente en el manejo de heridas en animales silvestres, particularmente tortugas y aves (Mickelson et al, 2016), ya sea porque promueve el proceso de cicatrización como también por sus propiedades antibacterianas (Mickelson et al, 2016; Scheelings \& Hellebuyck, 2019). El azúcar (sacarosa) y la miel han sido utilizadas desde antes de la era cristiana para la cicatrización de heridas en seres humanos. Actualmente son empleadas en todo el mundo para tratar heridas contaminadas y lentamente están empezando a tener aceptación en medicina veterinaria. A pesar de los excelentes resultados obtenidos, el mecanismo de acción de estas sustancias recién fue dilucidado en el siglo XX (Pintos, 2015).

\section{Presentación del caso}

Se presentó al consultorio un ejemplar de tortuga argentina, C. chilensis, macho, adulto (edad exacta desconocida), con un peso de 590 gramos y condición corporal 3/5. La propietaria refirió que el paciente había sido atacado por un canino doméstico, al menos 24 horas antes de la consulta. A la inspección, se constató la fractura del plastrón, con pérdida del segmento frontal, siguiendo el borde entre las placas humerales y pectorales. Se observó la exposición de los músculos deltoideus, en su porción ventral, y supracoracoideus, ambos con aspecto seco y olor rancio (Figura 1).

Como primer paso se procedió a la contención química del paciente mediante anestesia general, utilizando un anestésico disociativo (6o mg/kg de ketamina) combinado con una benzodiazepina ( $1 \mathrm{mg} / \mathrm{kg}$ de diazepam) (Carpenter, 2018) aplicado por vía intramuscular debido a que el animal se encontraba retraído en el caparazón. Una vez relajado el paciente, se colocó un acceso venoso en la vena yugular derecha con un catéter $24 \mathrm{G}$, previa antisepsia con alcohol y se procedió a su hidratación con suero Ringer lactato a razón de $30 \mathrm{ml} / \mathrm{kg} / \mathrm{día}$, durante 48 horas. Se realizó una limpieza profusa con clorhexidina al 2 \% y solución fisiológica, removiendo con un cepillo suave los contaminantes superficiales. 


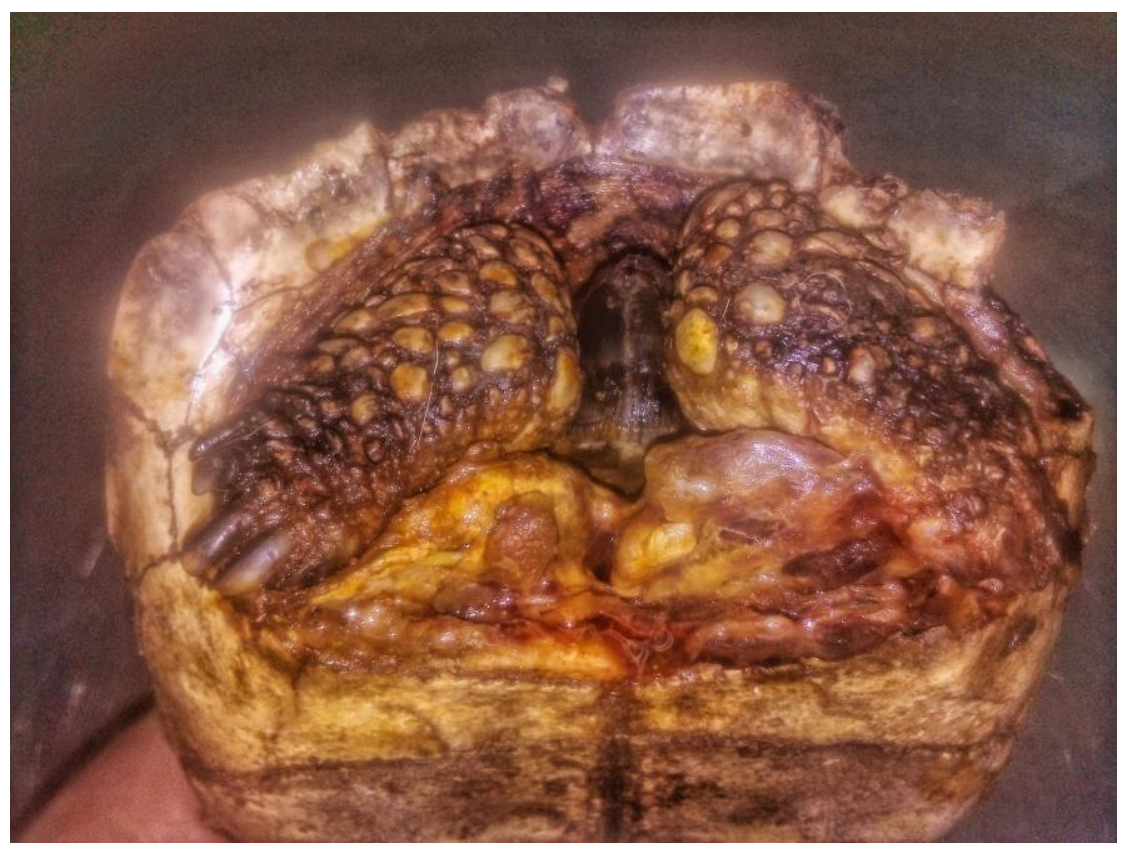

Figura 1. Lesión que evidencia la fractura del segmento frontal del plastrón con exposición muscular, en una tortuga argentina (Chelonoidis chilensis) mordida por un canino.

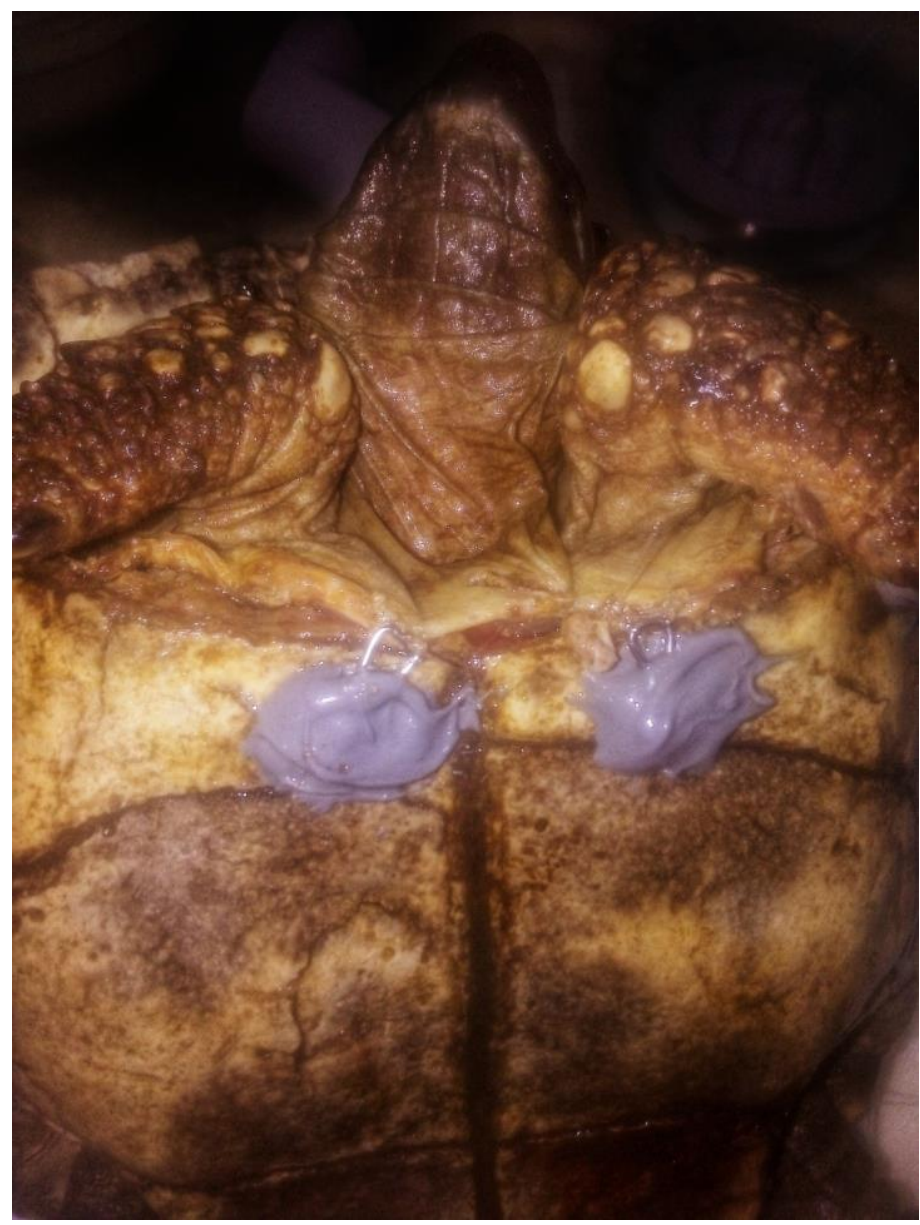

Figura 2. Utilización de ganchos fijados al plastrón para realizar una sutura de aproximación en una tortuga argentina (Chelonoidis chilensis) mordida por un canino. 
Se fijaron 3 ganchos al plastrón con resina epoxi, buscando puntos intermedios en donde fijar la piel. Hacia craneal de cada gancho se realizaron suturas de aproximación con poliamida 0,30 entre la piel y los ganchos, tratando de evitar una tensión excesiva entre los mismos (Figura 2). Se colocó una sonda de esofagostomía, utilizando una sonda nasogástrica K 35 fijada al caparazón mediante sutura en sandalia romana y material adherente.

Se instauró tratamiento antibiótico (22 $\mathrm{mg} / \mathrm{kg}$ de cefazolina) por vía intramuscular, cada 24 horas durante 10 días (Carpenter, 2018). Los espacios abiertos entre piel y plastrón fueron cubiertos con miel de abeja en forma tópica. En principio se realizó la aplicación de miel cada 48 horas; sin embargo, como al día 5 de tratamiento se percibió el crecimiento de Fusarium spp. en la superficie intervenida, se comenzó a realizar diariamente el lavado de la zona con iodopovidona al 7,5 \% previamente a la aplicación de miel.

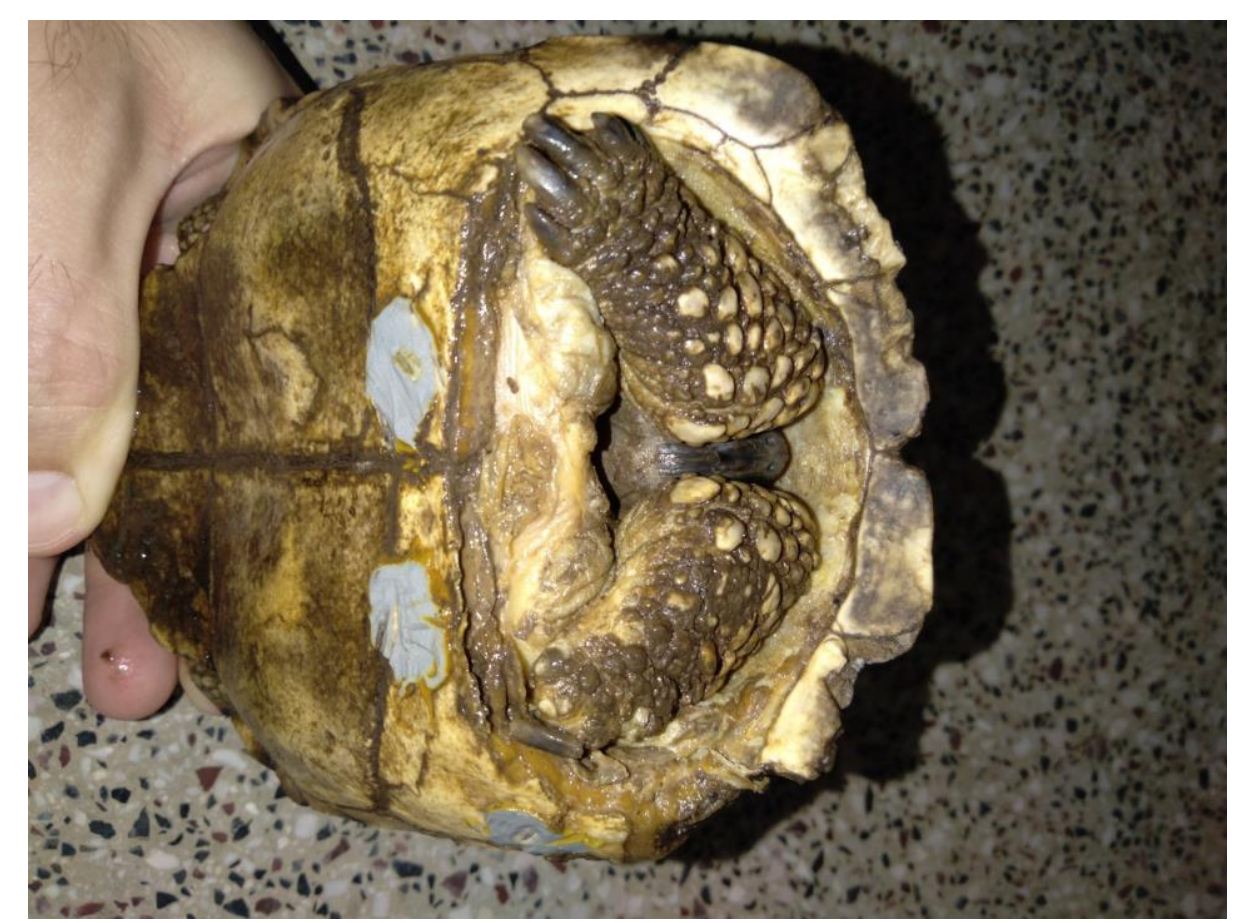

Figura 3. Tegumento remodelado de una tortuga argentina (Chelonoidis chilensis) mordida por un canino. El traumatismo fue resuelto mediante aproximación de piel y utilización de miel de abeja.

La alimentación del paciente por sonda fue realizada sobre la base de la Tasa Metabólica de Mantenimiento (TMM) en tortugas terrestres, utilizando la siguiente fórmula: $\mathrm{TMM}=\mathrm{K} \times \mathrm{Po}, 75$, siendo $\mathrm{K}$ la constante (32 en tortugas) y $\mathrm{P}$ el peso del animal (Pereira Dutra, 2014). Se administraron $20 \mathrm{Kcal} /$ día.

A las 48 horas del procedimiento se retiró el catéter intravenoso. El paciente fue colocado en baños de agua durante 2 horas, cada 48 horas. A los 5 días posteriores al procedimiento, comenzó a ingerir agua, y a los 35 días empezó a alimentarse por cuenta propia. A los 45 días se retiró la sonda esofágica. El paciente fue mantenido a una temperatura promedio de $30^{\circ} \mathrm{C}$ durante el día y $25{ }^{\circ} \mathrm{C}$ durante la noche, sobre una superficie de papel de diario durante 45 días 
y posteriormente sobre tierra. Si bien se le ofrecieron varios alimentos, solo seleccionó y se alimentó de zapallo hervido (Cucurbita spp) y nopal (Opuntia spp).

A los 10 días se observó la desecación de la piel que cubría el borde libre del hueso, no así la piel que recubría los músculos. A los 28 días se percibió buena adherencia de la piel a los músculos, al tiempo que, coincidentemente, empezó a utilizar los miembros anteriores. A las 6 semanas se retiraron las suturas y se constataron una remodelación del tegumento y un cierre completo de la herida (Figura 3). Transcurrido un año desde el inicio del tratamiento, la retracción cicatricial en el sitio de la lesión no produjo consecuencias para el normal desenvolvimiento del animal.

\section{Discusión y conclusiones}

El tiempo de resolución de las heridas en tortugas varía dependiendo del estado nutricional, la temperatura ambiental, la presencia de cuerpos extraños o fluidos en la herida, y la posible infección (Mickelson, 2016). En general, se menciona un tiempo de cicatrización de entre 6 y 8 semanas antes de retirar las suturas (Negrini et al, 2016; Scheelings \& Hellebuyck, 2019).

La contaminación con Fusarium spp. puede deberse a diversos motivos. Según Paterson (2006), el hongo es un saprofito ambiental común, ocasionando una enfermedad de progresión lenta que rara vez compromete la vida del animal. Por otro lado, también se ha demostrado la presencia del hongo en la miel de abeja (Grabowski \& Klein, 2017; Kačániová et al, 2012). Es decir que cualquiera de los dos motivos, o ambos, podrían haber sido la causa del desarrollo de este hongo en el paciente tratado.

En cuanto a la miel de abeja utilizada dentro del estudio, Pintos (2015) describió que el azúcar y la miel crean un medio con bajo contenido de agua (alta osmolaridad), que genera migración de agua y linfa fuera del tejido, hacia la solución de azúcar, inhibiendo el crecimiento bacteriano por disminución de la "actividad de agua" (Aw) del sustrato, mientras la linfa continúa suministrando nutrientes al tejido. La "Aw" es la relación entre la presión de vapor del agua de un sustrato en relación con la presión del vapor de agua pura a la misma temperatura. En otras palabras, es la medida del agua disponible que existe en un sustrato y depende del tipo y cantidad de interacciones del agua con otros componentes de dicho sustrato. La Aw influye en el crecimiento, la resistencia y la supervivencia de los microorganismos. Además, atraen macrófagos, que participan en la "limpieza de la herida", acelerando el desprendimiento de tejido desvitalizado, necrótico y/o gangrenoso, aportando una fuente de energía local y formando una capa proteica protectora en la herida. No solo absorben líquidos del citoplasma de las bacterias, sino de las células superficiales del lecho de la herida. Tales células superficiales, al contrario de las bacterias, están conectadas unas con otras, por lo que no se produce deshidratación de estas, sino una migración de líquidos y sangre de los sustratos profundos a la superficie de la herida, con formación de microcapilares. Así, con una nutrición y humedad adecuadas se favorece el desbridamiento, evitando los malos olores al acabar con los esfacelos y el sustrato bacteriano, generando una cicatrización más rápida, limpia, y consolidada. La concentración de macrófagos acumulados en la 
lesión favorece un aumento de los fibroblastos e incentiva la producción de colágeno, por lo cual, al cabo de una semana, aparecerá el tejido de granulación (Pintos et al, 2015).

Si bien se intentó emplear apósitos autolíticos, como alginato de calcio, no se encontraron en el mercado.

\section{Declaración de conflicto de intereses}

No existe conflicto de intereses, incluyendo entre estos últimos las relaciones financieras, personales o de otro tipo con otras personas u organizaciones que pudieran influir de manera inapropiada en el trabajo.

\section{Agradecimientos}

Milagros Martínez, Elfriede Hiebert, Rigoberto García, Dalila López. El trabajo fue financiado con medios propios de los autores.

\section{Referencias}

Cabrera MR. 1998. Las tortugas continentales de Sudamérica Austral. Córdoba, edición privada del autor.

Carpenter JW, Marion CJ. 2018. Exotic animal formulary. $5^{\circ}$ ed. St. Louis, Elsevier.

Grabowski NT, Klein G. 2017. Microbiology and foodborne pathogens in honey. Critical Reviews in Food Science and Nutrition. 57(9):1852-62. https://doi.org/10.1080/10408398.2015.1029041

Gray JE. 1870. Note on Testudo chilensis. Annals and Magazine of Natural History. 6(4):428.

Kačániová M, Kňazovická V, Felšöciová S, Rovná K. 2012. Microscopic fungi recovered from honey and their toxinogenity. Journal of Environmental Science and Health. Part A, Toxic/Hazardous Substances \& Environmental Engineering. 47(11):1659-64. https://doi.org/10.1080/10934529.2012.687242

Mickelson M, Mans C, Colopy S. 2016. Principles of wound management and wound healing in the exotic pets. Veterinary Clinics of North America: Exotic Animal Practice. 19(1):33-53. https://doi.org/10.1016/j.cvex.20 15.08.002

Negrini J, Ginel P, Novales M, Guerra R, Mozos E. 2016. Clinical and histological findings of cutaneous wound healing in the red-eared slider turtle (Trachemys scripta elegans) housed in unheated outdoor enclosures. Veterinary Dermatology. 27(5):413-e106.

https://doi.org/10.1111/vde.12346

Paterson S. 2006. Skin diseases of exotic pets. Oxford, Blackwell.

Pereira Dutra GH. Testudines. En: Cubas ZS, Silva JCR, Catao-Dias JL. 2014. Tratado de animais selvagens. $2^{\circ}$ ed. Sao Paulo, Roca, pp. 219-58.

Pintos S, González R, González E. 2015. Cicatrización de injertos cutáneos en malla de espesor completo utilizando azúcar granulado versus nitrofurazona en caninos. Revista Veterinaria. 26(2):103-07.

Romero F, Espinoza A, Salaberry-Pincheira N, Napolitano C. 2019. A five-year retrospective study on patterns of casuistry and insights on the current status of wildlife rescue and rehabilitation centers in Chile. Revista Chilena de Historia Natural. 92(6):1-10. https://doi.org/10.1186/s40693-019-0086-0

Scheelings T, Hellebuyck T. Integument. En: Divers S, Stahl S. 2019. Mader's reptile and amphibian medicine and surgery. St. Louis, Elsevier, pp. 1042-43.

Van Dijk PP, Iverson J, Rhodin A, Schaffer B, Bour R. 2014. Turtles of the world, 7th edition: Annotated checklist of taxonomy, synonymy, distribution with maps, and conservation status. Chelonian Research Monographs. 5(7):329-479. 
Vetter Hiebert JR, Riveros Núñez EH. 2021. Control de roedores sinantrópicos por ataques a tortugas terrestres, Chelonoidis carbonaria (Spix) y Chelonoidis chilensis (Gray), juveniles, en cautiverio. Boletín del Museo Nacional de Historia Natural del Paraguay. 25(2): 89-100. 